\title{
La interacción paciente-dentista, a partir del significado psicológico de la boca
}

\author{
Rojas Alcayaga G*, Misrachi Launert C**
}

\section{RESUMEN}

La boca es una parte integrante no sólo de un cuerpo físico, sino también de una imagen corporal o dicho en otros términos, de un cuerpo representado a nivel psíquico. Es así que posee un significado psíquico único en relación con otras partes del cuerpo. Debido a las experiencias primarias que la boca permite en el desarrollo humano, es que en ella están representados sentimientos íntimamente ligados a la identidad del sí mismo. Cuando el dentista se relaciona con su paciente deberá considerar estos conceptos para conseguir una buena alianza terapéutica. La relación se verá fortalecida en la medida que se considere al paciente como una persona independiente que tiene ciertos conocimientos y capacidad de decisión respecto a su salud oral. Si esto se complementa con la aplicación de una serie de técnicas que ayudan a disminuir la ansiedad del paciente cuando asiste a la consulta profesional, se logrará que desarrolle conductas de autocuidado para mejorar y mantener su salud bucal en buenas condiciones.

Palabras clave: relación dentista-paciente, estrés en odontología, ansiedad dental, ciencias del comportamiento en odontología.

\begin{abstract}
The mouth is not only a part of the physical body, but also a part of the body image present at the psychical level. In relation to others parts of the body, the mouth has a unique psychological meaning. Feelings represented in the mouth are closely bonded to the self identity, because of the primary experiences transmitted by this part of the body. The dentist must consider these concepts while retreating to this patient, so as to succeed in a adequate therapeutic alliance. This relation will become stronger by means of considering the patient as an independent person who has certain baggage of knowledge and capacity over his/her oral health.

All this is complement with a series of procedures to help diminish the patient's anxiety produced every time that he/she attains dental practice.
\end{abstract}

Key words: Dentist-patient interaction, stress in dentistry, dental anxiety, behavioral dental science.

* Cirujano Dentista. Magíster en Ciencias Odontológicas, Univ. De Chile. Licenciado en Psicología, Univ. de Chile. Prof. Asistente. Facultad de Odontología. Universidad de Chile. Área Ciencias del Comportamiento.

** Cirujano Dentista. Magíster en Educación Pontificia Univ. Católica. Prof. Titular. Facultad de Odontología. Universidad de Chile. Área Ciencias del Comportamiento.

Rojas Alcayaga G, Misrachi Launert C. La interacción paciente-dentista, a partir del significado psicológico de la boca. Av. Odontoestomatol 2004; 20-5: 239-245.

\section{INTRODUCCION}

La visita al dentista es una de las situaciones que más ansiedad genera en las personas, llegando a veces a tal grado que el paciente deja de acudir a la consulta profesional abandonando los tratamientos. Es evidente que tal conducta atenta contra la salud oral de esas personas. Además el estado altamente 
ansioso del paciente influye notoriamente en la calidad de los tratamientos, así como también en la salud mental del odontólogo.

La manipulación de la zona bucal por parte del dentista, despierta en los pacientes, diversos grados de aprensiones y ansiedades. Es habitual que las personas señalen que la visita al dentista es una de las atenciones en salud que más miedo les provoca.

No está claro a que se debe que el miedo a la atención odontológica sea tan frecuente en la población, sin embargo la mayoría de las explicaciones apuntan a procesos psicológicos relacionados tanto con el aprendizaje de esa actitud, ya sea a nivel individual y/o social, como con el significado psicosocial que se le otorga a la boca.

\section{SIGNIFICADO PSICOSOCIAL DE LA BOCA}

No es desconocido que Freud asignó a la boca una función primordial en el desarrollo psicosexual de la persona. La boca constituye el primer órgano de contacto con el mundo y a través de ella el nuevo ser obtiene la primera experiencia del sí mismo, que indeleblemente permanecerá como núcleo central en la estructura de la personalidad futura. Freud la describió como la fase oral, la cual es seguida por la fase anal y posteriormente por la fase genital. Esta fase oral dura aproximadamente hasta el año y medio de edad y se caracteriza por el chupeteo de los objetos, como modo de conocimiento del mundo externo (1).

La boca es el asiento psicológico de las primeras necesidades fisiológicas y gratificaciones emocionales, con ella se toma el gusto al mundo que nos rodea. La boca provee las primeras sensaciones de seguridad, placer, satisfacción y éxito, llevando toda esta actividad oral a las primeras percepciones de sí mismo (2).

En la fase oral la boca es fuente de placer al realizar el acto de succión del pezón materno, en una primera fase el pecho de la madre se percibe como indiferenciado de sí mismo pues el lactante percibe que él y su entorno son una misma cosa. Sin embargo, en un momento posterior el niño constatará que el pecho materno está separado de él, generando así la primera frustración y la primera percepción de un mundo diferenciado de él mismo. Por lo tanto esta experiencia, relación niño-pecho materno, implica un modo de relación con el mundo externo, físico y social. Por lo mismo cualquier disturbio en esta fase, ya sea por exceso o falta de gratificación, se relacionará con un modo poco adaptado de "ser en el mundo". Así en personas con fijaciones en la fase oral, la zona bucal queda revestida de una carga emocional excesivamente elevada, llevándolos a necesidades de satisfacción inacabables. Estas personas se caracterizarán por constantes quejas, nauseas y asco. Emocionalmente son personas exigentes, dominantes, codiciosas o dependientes (2).

Más allá de la presencia de alteraciones del desarrollo en la fase oral, lo relevante es comprender el fuerte significado psicosocial que tiene la zona bucal para el ser humano, de alguna forma en ella estamos íntegramente representados y se devela nuestra intimidad. Al respecto debemos recordar el concepto de esquema o imagen corporal, que corresponde a la vivencia psíquica del cuerpo, la que otorga una representación de integridad del yo. En relación a esto, es interesante señalar el fenómeno del miembro fantasma, en el cual la persona sigue percibiendo una parte del cuerpo que ha sido amputada. La concepción que tienen los individuos sobre la imagen del mismo está mucho más en función de la calidad de la investidura libidinal de este cuerpo que de la realidad, por ejemplo las pacientes anoréxicas se perciben efectivamente como obesas, aún cuando estén objetivamente enflaquecidas. Así podemos ver que junto a la realidad de la anatomía objetiva, necesitamos reconocer la realidad de una "anatomía del deseo" (3). La boca desempeña una función importante en el sentimiento de unidad y en la constitución del yo, el funcionamiento oral sirve de modelo corporal sobre el cual se apoyan las vivencias psíquicas que acompañan al proceso de identificación, por lo tanto recibe importantes cargas libidinales. Es decir en la boca está representado en forma importante el "yo", pues marca claramente un límite entre el adentro y el afuera (tal cual también lo hace la piel) y se constituye en un elemento de intercambio con el mundo externo (la alimentación, el habla, el gusto, 
etc.), la boca individualiza, hace reconocible lo propio de lo ajeno, en la boca estamos representados íntegramente. Por lo mismo es comprensible la dificultad de exponer esta zona del cuerpo tan íntima a otra persona como lo es el odontólogo, que en muchos casos es un desconocido y que además va a realizar intervenciones que tanto objetivamente como subjetivamente implican alguna forma de agresión. No es tan sólo el daño físico, es la amenaza a la integridad psicológica, a la percepción de sí mismo.

\section{EXPLICACIONES DE LA ADQUISICIÓN DEL MIEDO DENTAL}

No existe una respuesta única ni definitoria para explicar este fenómeno, pero al parecer en la mayoría de los casos esta actitud es debida a experiencias con el dentista cuya significación resulta traumática para el paciente. Los trabajos de Molin y Seeman, citados en Scott y Hirschman (4), señalan que los sujetos encuestados reportan que sus experiencias con el dentista contribuyen significativamente en la generación de ansiedad dental. Estas personas declaraban que; la creencia de haber recibido un tratamiento inapropiado y el manejo brusco por parte del profesional, eran los factores que más contribuían al desarrollo de ansiedad frente al tratamiento odontológico.

En el mismo artículo se discute la posibilidad de que la alta ansiedad tenga que ver con una mayor sensibilidad a los procedimientos dolorosos. De este modo, una de las hipótesis que se maneja es que esos pacientes son hipersensibles al dolor en general, sin embargo otros estudios citados por Scott y Hirschman (4) indican que la baja tolerancia al dolor es específica al tratamiento dental. Por otro lado algunos investigadores han propuesto que la ansiedad dental es sólo una faceta de una alta ansiedad generalizada la que se manifiesta de diversas maneras. Otras hipótesis apuntan a que esta ansiedad se origina por transmisión padres-hijos de actitudes desfavorables hacia el dentista como también por aprendizaje social, a través de las interacciones con otras personas que relatan historias de horror respecto al tratamiento dental. (4)
$\mathrm{Al}$ respecto Gale (5) diseñó una escala que le permitió evaluar situaciones relacionadas a la atención dental que causaban más miedo a los pacientes. Al hacer un ranking de las aseveraciones que recibieron más puntaje, resultaron como las más de temer ; la extracción dentaria, el fresado dental y una mala opinión de los dentistas respecto a las condiciones de salud oral del paciente, superando esta última al hecho de sostener la jeringa con la aguja expuesta delante del paciente. Gale señala que se debe tener muy en cuenta que la actitud del dentista hacia el paciente condiciona mucho la actitud de éste hacia el cuidado de la salud bucal y hacia el dentista en particular y al respecto señala que una critica hacia las condiciones de la boca es percibida por el paciente como una crítica al "sí mismo".

\section{EL ESTRÉS EN LA ATENCIÓN ODONTOLÓGICA}

En todo caso es indudable que para muchas personas la atención dental es una situación estresante. Al respecto es conveniente recordar la distinción entre estrés fisiológico y psicológico, siendo el primero una respuesta orgánica a un daño tisular real (por ejemplo una extracción dentaria) y el segundo una respuesta a una amenaza simbólica. Sin embargo el estrés psicológico puede connotar consecuencias fisiológicas idénticas a la del estrés fisiológico (6).

El estrés psicológico implica siempre un problema de anticipación del futuro, la gente se estresa por lo que va a pasar, no tanto por lo que está pasando en el momento mismo. Evidentemente ese futuro aparecerá como amenazante para la persona. Lazarus (6) diseñó una experiencia en la cual los sujetos eran sometidos a distintos tipos de inyecciones anestésicas, desde unas que consistían en la inyección de anestésicos con vasoconstrictor hasta otras en que sólo se colocaba la jeringa con la aguja dentro de la boca del paciente, sin realizar punción alguna. Lo interesante es que independiente de la situación, las personas desarrollaron un semejante patrón de respuestas fisiológicas indicadoras de estrés (medido por reacción electrodérmica). Esto demuestra que la respuesta de estrés no es al daño mismo sino a la percepción subjetiva de amenaza. A esto contribuyen 
dos factores; uno relacionado al tiempo de anticipación y el otro a lo que se piensa o interpreta de la situación estresante. En general se ha observado que mientras más tiempo transcurre entre la noticia de exposición al estímulo y la exposición misma, mayor es el nivel de estrés. Por otro lado el nivel de estrés se relaciona directamente con la evaluación cognitiva que haga la persona de la situación en cuestión, es decir, de cómo interpreta el estímulo (7). Al respecto en un experimento también conducido por Lazarus (6) se observó que en la observación de una película de fuerte contenido traumático, la reacción de las personas dependía en gran parte de la explicación que se daba del suceso, concluyendo que en la medida que se intelectualizaba o se negaba el sufrimiento de la persona se reducía el nivel de estrés de los observadores de la película. Por todo lo anterior es interesante señalar que el desarrollo de estrés depende en gran parte de las características del individuo, principalmente de las ideas que despliega en el enfrentamiento de la situación aversiva (7). Estas ideas (esquemas cognitivos) justamente provienen de experiencias anteriores que han modelado una forma de ver y ser en el mundo, lo interesante es buscar métodos que modifiquen esos esquemas de pensamiento cuando se refieren a procesos dentales vistos como amenazantes.

\section{LA RELACIÓN DENTISTA - PACIENTE}

Por otro lado es relevante evaluar el significado que reviste la relación paciente-dentista. Se añade a la significación oral previamente descrita, una relación que simbólicamente representa las primeras experiencias con la autoridad, ya sea el padre o algún otro significativo.

Del momento que el paciente elige a un determinado odontólogo ya está poniendo en juego su actitud hacia las figuras significativas. Cuando ingresa al consultorio dental lleva consigo toda una forma de reaccionar frente a los procedimientos odontológicos que comprometen su boca, como al dentista, en cuanto figura revestida de autoridad.

El paciente llega al dentista con sentimientos contradictorios. Por un lado lo percibe, al igual que en toda la profesión médica, como un ser dotado de poderes (reales y fantasiosos) que le permitirá aliviar sus dolencias. Sin embargo es a la vez una figura amenazadora, dado los procedimientos invasivos y eventualmente dolorosos aplicados en la zona bucal. El paciente sufre además otro conflicto, sabe que necesita de tratamiento odontológico pero al mismo tiempo percibe la situación como amenazadora. Este complejo emocional lleva al paciente a un estado de ansiedad, a constantes interrupciones al accionar del dentista, postergaciones de las citas o la suspensión del tratamiento (2).

Es responsabilidad del odontólogo intervenir para disminuir ese estado ansioso, para conseguirlo es fundamental el estilo interpersonal en el cual se desarrolle la relación paciente-dentista. Como primer paso el dentista debe considerar la condición psicológica del paciente, pues éste de alguna manera sufre una regresión infantil, se hace muy dependiente de su dentista en el momento de la atención odontológica y se reeditan las primeras relaciones con figuras de autoridad. El dentista dispone de un poder inmensamente superior al de su paciente cuando éste se encuentra en el sillón dental, pues simbólicamente se produce nuevamente una restitución de la dinámica padre - hijo.

Es en la primera consulta donde se despliegan los sentimientos del paciente respecto al tratamiento odontológico y al dentista. Esta es la oportunidad para que el profesional intervenga para proveer un ambiente psicológico de seguridad, que permita develar temores y ansiedades propias a la situación de atención dental.

El cuidado de la salud oral es un esfuerzo de dos personas, no el trabajo de una persona sobre la otra. Para tal efecto el dentista debe ser flexible cuando deba hacer modificaciones en el plan de tratamiento cada vez que surja alguna necesidad particular en el paciente respecto al mismo. De esta manera sí se maximiza el estatus de igualdad dentista/paciente, al mismo tiempo se minimiza la posibilidad de disrupciones dentro de la interacción. En este sentido es vital comprender que dicha relación está conformada por dos adultos, la cual es única e igualitaria, así se posibilita una interacción realista y no fantasiosa, una relación de este tipo no es afectada por la ansie- 
dad o la preocupación del paciente acerca del tratamiento dental (8).

Se debe buscar una alianza de tratamiento, es decir paciente y dentista se deben alinear en un objetivo común, en este sentido la ansiedad dental es una barrera para tal alianza. Sin embargo el dentista junto al paciente deben abordar las fuentes de esta ansiedad de manera tal de que, más que una interferencia sea una guía para la planificación del tratamiento a seguir, así la tarea de delinear un tratamiento es de común acuerdo, se fortalece así la alianza terapéutica en vez de debilitarse a causa de la ansiedad del paciente.

La transferencia (fenómeno psicológico que hace referencia a la reedición de pautas de comportamiento interpersonal aprendidas en periodos precoces de la vida y que se ponen en juego en cada relación posterior con un otro significativo) debe ser considerada en la atención dental. El paciente en la atención dental sufre una regresión a una etapa infantil y desde esa condición emocional se relaciona con el dentista y reactualiza la regresión a la relación padre-hijo. Dada la relación de autoridad, visualiza al dentista en el rol de padre. Es así como el dentista puede ser percibido como un padre protector ó extremadamente autoritario con gran capacidad de generar miedo o daño (8).

Szasz y Hollender (8) propusieron tres modelos básicos en la relación paciente/dentista; 1) Actividadpasividad, 2) Guía-cooperación y 3) Participación mutua. En la primera el dentista le hace algo al paciente, el que a su vez sólo cumple con el rol de recibir el tratamiento, en este modelo se reconstituye la dinámica padre-hijo. En el segundo el dentista le dice al paciente lo que le va a hacer, señalando éste su acuerdo, nuevamente se establece una relación padre-hijo. En la tercera el dentista le sugiere y negocia con el paciente el tratamiento a realizar, en una clara relación entre adultos de igual condición. En esta última modalidad el dentista reconoce el potencial del paciente y comprende que éste no es un ignorante respecto a lo que se le debe hacer como tratamiento, sino que siempre sabe algo. En esta interacción la alianza de tratamiento se fortalece, favoreciendo en definitiva una mayor responsabilidad del paciente sobre su propia salud oral.
Un estudio realizado por Peñaranda (9) determinó, mediante una encuesta hecha a dentistas, las aplicaciones en la odontología de conocimientos de psicología. De una diversidad de temáticas resultó que el manejo de niños con problemas conductuales, los pacientes ansiosos, el cobro de los honorarios y los individuos con problemas de personalidad eran áreas donde el conocimiento de técnicas psicológicas resultó de gran utilidad para un mejor manejo de dichas situaciones. Sin embargo, el autor reconoce que en casi todas las acciones odontológicas son necesarios conocimientos y destrezas del ámbito de la psicología, pues en definitiva el éxito del tratamiento en gran parte depende de la actitud, motivación, y conducta del paciente con relación al tratamiento. Además, no se debe olvidar las consecuencias que sobre el ánimo y conducta del profesional tienen las acciones desarrolladas por el mismo (ejemplo; cobro de honorarios), como también las desarrolladas por el paciente. En definitiva, un buen manejo psicológico del paciente redunda tanto en una mejor calidad y pronóstico del tratamiento, como en una mejor calidad de vida para el odontólogo.

\section{EVALUACIÓN Y TRATAMIENTO DEL MIEDO DENTAL}

Uno de los propósitos en la investigación de las ciencias del comportamiento en odontología ha sido el desarrollo de metodologías que permitan medir la ansiedad frente a la visita al dentista. El principal instrumento desarrollado son los cuestionarios. Uno de los primeros descritos es la "Escala de Ansiedad Dental (EAD)" desarrollado por Norman Corah en el año 1969. Es un instrumento sencillo, consta de 4 preguntas de autoadministración y sus respuestas son de alternativas, tipo Likert. Dispone de una alta validez y confiabilidad pese al reducido número de itemes. Los autores señalan que esta validez y confiabilidad se debe a que el cuestionario está libre de respuestas prejuiciadas, ya que tener miedo al dentista es socialmente aceptado, por lo cual el que responde la prueba no trata de buscar la respuesta adecuada sino que responde honestamente (10). Otros investigadores como Weinstein, citado por Corah 1978 (11), confirmaron la validez de esta prueba. 
Utilizaron la misma prueba en una muestra de 751 pacientes, reportando que altos puntajes en la EAD se asocian con insatisfacción de los pacientes (medida a través de una cuestionario) y concluyen que pacientes altamente ansiosos al tratamiento odontológico tienen una actitud negativa hacia el mismo, transformándolos en pacientes dificultosos. Auerbach, citado por Corah (11), administró la EAD a 63 pacientes, días antes de ser sometidos a cirugía bucal, el puntaje en la prueba fue capaz de predecir el estado de ansiedad mostrado por los , pacientes durante el procedimiento quirúrgico. Por último Weisenberg, también citado por Corah (11). aplicó la prueba a 72 pacientes de servicios de urgencia, y reportó una interrelación significativa entre la EAD y el Inventario de Rasgos de Estado de Ansiedad. Todos estos estudios proveen de sustrato para la validez predictiva de la prueba EAD. Corah (11) señala que los puntajes en la EAD son más altos en mujeres que en hombres, sin embargo no pueden determinar con certeza si las diferencias observadas son el resultado de diferencias en ansiedad o representan sesgos al momento de reportar la ansiedad. En este mismo estudio y como otra evidencia de la validez de la $E A D$, se administró esta prueba a pacientes con fobia dental, antes y después de recibir tratamiento para sus fobias a partir de procedimientos de desensibilización sistemática. Los resultados muestran una evidente disminución en los puntajes de la prueba concordante con un cambio de conducta hacia el tratamiento dental, por parte de los pacientes.

Corah y col. (12) señalan que la ansiedad del paciente es una variable que interfiere significativamente en la calidad de la atención dental y plantean la necesidad de aplicar estrategias para su control. Ellos separan las intervenciones posibles en tres categorías: relajación, distracción y percepción de control de estímulos. Para evaluar cada una de ellas diseñaron un experimento en el cual dispusieron de tres grupos, uno control, uno sometido a técnicas de relajación, y otro a un método de distracción; todos sometidos a un mismo tratamiento dental (restauraciones de amalgama). La evaluación de la ansiedad se realizó con la escala EAD como también con un dispositivo para registrar la respuesta electrodérmica durante la sesión. Concluyeron que la mayor reducción en la ansiedad se observa en el grupo sometido a relajación, con mayor respuesta de las mujeres que de los hombres. La distracción también funcionó en la reducción de la ansiedad principalmente en los hombres. El mismo autor en un estudio previo evaluó la percepción de control mediante el uso de un dispositivo que permitía al paciente señalar la interrupción del procedimiento dental, sin embargo no encontró diferencias significativas en el disconfort que originaba la atención dental al paciente, entre grupos en que se aplicó la estrategia y grupos control. Por ello concluyó que la percepción de control no constituía un método adecuado para disminuir la ansiedad de los pacientes con miedo dental (13).

\section{CONCLUSIÓN}

En la interacción dentista-paciente intervienen una serie de variables que determinan que esta relación represente simbólicamente el encuentro entre dos personas, una de las cuales dispone de un poder superior a la otra. Es así como se generan fenómenos de dependencia y/o de ansiedad, lo que claramente dificulta la toma de conciencia del paciente de su responsabilidad en el cuidado de su salud oral. Por lo tanto la forma que adopte esta relación tiene implicancias no sólo en la atención individual, sino repercusiones a nivel de salud pública. Es necesario seguir desarrollando investigaciones que aumenten el conocimiento en esta área para disponer de mejores herramientas para reorienten la dinámica dentista-paciente de modo de favorecer un mejor nivel de salud oral en la población.

\section{BIBLIOGRAFÍA}

1. Fadiman, J. y Frager, R. "Teorías de la Personalidad". Cap. 1. Oxford University Press México. México. 1976.

2. Ewen, S.J. "Psicología de las Enfermedades Orgánicas". Cap. 14. Editorial Paidos. Buenos Aires, Argentina. 1977.

3. Jeammet, P.; Reynaud,M. y Consoli, S.M. "Psicología Médica". Editorial Masson. $2^{\circ}$ edición. Barcelona. 1999. 
4. Scott, D. y Hirschman, R. "Psychological aspects of dental anxiety in adults". J. American Dent. Assoc. 104: 27-31.

5. Gale,E. "Fears of the dental situation". J. Dent. Res. Vol.51: 964-6. 1972.

6. Lazarus, R. y Folkman, S. "Estrés y Procesos Cognitivos". Capítulo 1. Editorial Martínez Roca. Barcelona, España. 1986.

7. Lazarus,R. "Some principles of Psychological Stress and their relation to dentistry". J. Dent.Res. vol.45:1620-6. 1966.

8. Freeman, Ruth. "A psychodynamic understanding of the dentist-patient interaction". British Dental Journal. Vol. 186. No 10, 1999.

9. Peñaranda,P. "Importancia de la psicología en la Odontología”. Acta Odontológica Venezolana. Vol. 28: 3-11. 1990.

10. Corah,N. "Development of a dental anxiety scale". J. Dent. Res. Vol. 48: 596. 1969.
11. Corah,N. Gale,E. Y Illig,S. "Assessment of a dental anxiety scale". J. Am. Dent.Assoc. Vol. 97: 816-9. 1978.

12. Corah,N.; Gale,E, e Illig,S. "Psychological Stress Reduction During Dental procedures". J. Dent.Res. Vol.58: 1347-51. 1979.

13. Corah,N.; Bissell,D. E Illig,S. " Effect of Perceived Control on Stress Reduction in Adult Dental Patients". J. Dent.Res. Vol 57:74-6. 1978.

\section{CORRESPONDENCIA}

Facultad de Odontología.

Universidad de Chile. Casilla 1903.

Santiago de Chile.

Teléfono 56-2-6785039

Fax 7776062 